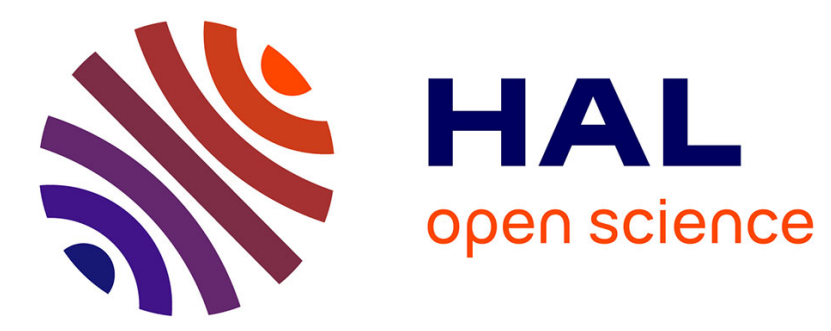

\title{
Improving Mobile IPv6 Handover in Wireless Network with E-HCF
}

Anne Wei, Gouzhi Wei, Benoit Geller

\section{To cite this version:}

Anne Wei, Gouzhi Wei, Benoit Geller. Improving Mobile IPv6 Handover in Wireless Network with E-HCF. IEEE Vehicular Technology Conference, Sep 2008, Calgary, Canada. pp.5-9, 10.1109/VETECF.2008.294 . hal-01229104

HAL Id: hal-01229104

https://hal-ensta-paris.archives-ouvertes.fr/hal-01229104

Submitted on 16 Nov 2015

HAL is a multi-disciplinary open access archive for the deposit and dissemination of scientific research documents, whether they are published or not. The documents may come from teaching and research institutions in France or abroad, or from public or private research centers.
L'archive ouverte pluridisciplinaire HAL, est destinée au dépôt et à la diffusion de documents scientifiques de niveau recherche, publiés ou non, émanant des établissements d'enseignement et de recherche français ou étrangers, des laboratoires publics ou privés. 


\title{
Improving Mobile IPv6 Handover in Wireless Network with E-HCF
}

\author{
Anne Wei ${ }^{* \dagger}$, GouZhi Wei ${ }^{*}$ and Benoit Geller ${ }^{\ddagger \S}$ \\ *Université Paris XII, 61 avenue du Général de Gaulle, 94030 Créteil, France \\ ${ }^{\dagger}$ Conservatoire National des Arts et Métiers, 292, rue Saint-Martin, 75003,Paris,France \\ ‡SATIE - ENS Cachan, 61 avenue du Président Wilson, 94235 Cachan Cedex,France \\ §LEI - ENSTA, 32 boulevard Victor, 75015 Paris Cedex,France \\ Email: anne.wei@cnam.fr
}

\begin{abstract}
Mobile IP allows a mobile node to maintain a continuous connectivity to the Internet when moving from one access point to another. However, due to the link switching delay and to the Mobile IP handover operations, packets designated to mobile nodes can be delayed or lost during the handover period. This paper presents a new control function called Extended Handover Control Function $(E-H C F)$ in order to improve the handover performance in the context of Mobile IPv6 over wireless networks. With an analytical model and some OPNET simulations, we show in this paper that our solution allows to provide low latency, low packet loss to the standard handover of Mobile IPv6.
\end{abstract}

Index Terms-Mobile IPv6, Performance and Handover operations

\section{INTRODUCTION}

$\mathbf{T}$ HE need to keep an "everywhere and at any time" connection with Internet has been more and more demanded in recent years with the success of IEEE 802.11 and of IEEE 802.16 wireless networks standards. A growing number of $802.16 / 802.11$ based wireless networks has been deployed in campuses, hotels, airports and companies as access networks to the Internet. The mobility support has thus become one very hot research subject. However, the continuous Internet connectivity and the correct routing of packets were not guaranteed when users change their access points to Internet with classical protocols. To resolve these problems, the Mobile IPv4 (MIPv4) and Mobile IPv6 (MIPv6)protocols [1], [2] were respectively published by the Internet Engineering Task Force (IETF).

Based on MIPv6, the main standards by the IETF are the Hierarchical Mobile IPv6 (HMIPv6) and the Fast Handover for MIPv6 (FHMIPv6). HMIPv6 introduces a Mobility Anchor Point (MAP) who acts somehow like a local Home Agent $(H A)$ for the visiting Mobile Node $(M N)$. The concept of $M A P$ can limit the amount of signaling required outside the MAP's domain [5], [7]. FHMIPv6 [8] can reduce the packet loss by providing fast IP connectivity as soon as a new link at the Link Layer is established. The network uses a Link Layer trigger to launch either Pre-Registration or Post-Registration handover operations. Besides of these main proposals, there has been some approaches for providing the

This work was supported in part by the international project PRA-SIP under Grant SIP04-03. lossless handover and minimizing the handover delay [9][12], [14]. In [9], a Pre-Handover Signaling (PHS) protocol is proposed in order to support the triggering of a predictive handover and to allow the network to achieve accurate handover decisions by considering different constraints such as Quality-of-Service $(Q o S)$, user profile and mobile node service requirements. In [10], a Hierarchical Network-layer Mobility Management $(H N M M)$ framework is described in which an integrated IP-layer handover solution provides an optimized network connectivity. Also, a Competition based Soft Handover Management (CSHM) protocol [11] and a Multi-path Transmission Algorithm (MTA) [12] have been presented to decrease packet loss during a handover. Furthermore, the IEEE 802.11f standard including the Inter-Access Point Protocol $(I A P P)$ enables the Access Points $(A P S)$ to communicate with each other, so that the Mobile IPv6 handover is improved at the Link Layer [14].

The goal of this paper is to optimize the Mobile IPv6 handover procedure by using a new function named Extended Handover Control Function $(E-H C F)$. Based on our paper [3], the principle of the Handover Control Function $(H C F)$ is that, according to the mobile node's scanning result and the $\mathrm{HCF}$ router database, the $H C F$ router can both pre-decide a mobile node's new access point and a new IP address. So the mobile node can send Binding Update message when it is still connected with its previous access point. Contrarily to a standard MIPv6 handover for which the Detection Address Duplication $(D A D)$ deteriorates dramatically the handover latency (see below), the $H C F$ approach avoids any IP address collision without the use of $D A D$. In this context, we propose the $E-H C F$ which not only inherits of the advantages of the $H C F$, but also allows communications between some extra$H C F$ routers. Moreover, the $E-H C F$ can buffer the packets during the handover process in order to reduce the packet loss. The remainder of the paper is thus organized as follows: Section II presents our Extended Handover Control Function $(E-H C F)$ architecture and the associated operations. Section III deals with the performance of the $E-H C F$ handover in terms of handover latency and packet loss. Regarding the standard handover of MIPv6, Our numerical and simulation results show that the $E-H C F$ handover reduces significantly both the latency and the packet loss. Finally, some conclusion and future works are mentioned in Section IV. 


\section{EXTENDED HANDOVER CONTROL FUNCTION FOR MOBILE IPV6}

\section{A. E-HCF overview}

Generally speaking, a handover consists of a Link Layer handover and of a Network Layer handover. The Link Layer handover includes a Discovery phase (scanning the channels to discover an available Access Point), an Authentication phase, and a Re-association phase, whereas the Network Layer handover is concerned by a Router Discovery phase, a Detection Address Duplication $(D A D)$ phase, a Binding Update phase and a Binding Acknowledgement phase respectively. As displayed on Figure 1, the standard MIPv6 handover latency has been estimated to a maximum value of $1290 \mathrm{~ms}$ [7]. This long latency is not acceptable for real time applications such as video and audio. If we analyze each phase during the Network Layer handover (Router Discovery, $D A D$, Binding Update and Binding Acknowledgement), we can note that the $D A D$ latency costs almost $1000 \mathrm{~ms}$ and has a heavy weight on the global handover latency. As a result, in order to reduce the total handover latency, we now develop a procedure to avoid any $D A D$ operation during handover procedure.

\begin{tabular}{|c|c|c|c|c|}
\hline \multicolumn{1}{|c|}{ Link layer } & \multicolumn{3}{c|}{ Network layer } \\
\hline Level 2 handover & Router Discovery & DAD & Binding Update & Binding Acknowledgement \\
\hline
\end{tabular}

Fig. 1. Standard MIPv6 Latency

We introduce a local intelligent entity called Extended Handover Control Function $(E-H C F)$ which should be capable of controlling its attached Access Routers (ARs), Access Points $(A P s)$ and Mobile Nodes $(M N s)$. As shown on Figure 2, linked directly with its ARs, each $E-H C F$ router reserves beforehand a list of all available IP local addresses. The $E-H C F$ router also generates and updates periodically a second list which records the used ARs/APs/IP addresses. By comparing these two lists, the $E-H C F$ router can find a potential duplicate IP address (collision) in its domain. Then, this $E-H C F$ router can withdraw this potential duplicate IP address or can ask a concerned sub-node to change its IP address. In this way, the $E$ - $H C F$ router enables to insure an unique IP address to a $M N$ without DAD.

Furthermore, an $E-H C F$ router could exchange both some local information with its $\mathrm{ARs} / \mathrm{APs} / \mathrm{MNs}$ and some external information with other $E-H C F$ routers. To realize our $E$ $H C F$ proposal, we propose six new messages: MN Request (MNReq), MN Reply (MNRep), HCF Request (HCFReq), HCF Reply (HCFRep), Connection Established Information (CEInf) and Handover Finished Confirmation (HFCon) messages (for the detailed information about the formats of these messages see [15]).

For the mobile IPv6 protocol and IEEE 802.11/802.16 networks context, a $M N$ surveys periodically the received signal strength. When the signal strength drops below a predefined threshold, the $M N$ must discover and connect itself to a new available $A P$ for granting its communication with its

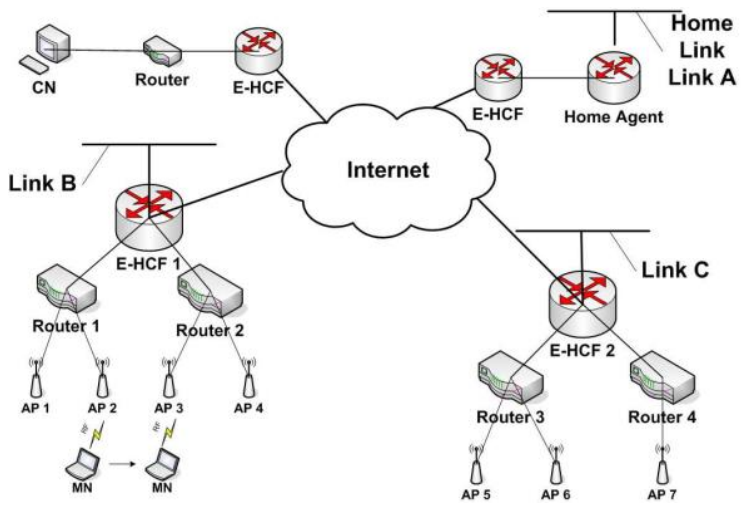

Fig. 2. Architecture of Extended Handover Control Function (Router is an Access Router; E-HCF is an E-HCF router)

correspondence. It reports to its $E-H C F$ router (via its attached $A R / A P$ ) some $A P$ 's Basic Service Set IDentifier (BSSID) and signal strengths that it were probed. Based upon the reported information, the $A R / A P$ 's loading and the $M N$ 's Quality of Service $(Q o S)$ requirements, the $E-H C F$ router decides which $A P$, the $M N$ shall associate with and notifies the $M N$ about the new $A R / A P$ information, such as a new AP's BSSID, an $A R$ interface address, a sub-network prefix and an IP address. Consequently, the $M N$ can configure its new Care-of-Address $(C o A)$ and can take care of the Binding Update process even if it is still attached with its previous $A R / A P$. An $E-H C F$ router can guarantee that the new IP address is unique thanks to the knowledge of its lists. If a $M N$ moves to another domain, the $E-H C F$ original router guarantees the new IP address by exchanging some data with the new $E-H C F$ router. Moreover, in order to minimize the packet loss during a handover, an $E$ $H C F$ router stores packets into a buffer until the $M N$ is really attached to the new IP address. The entire handover procedure is displayed on Figure 3.

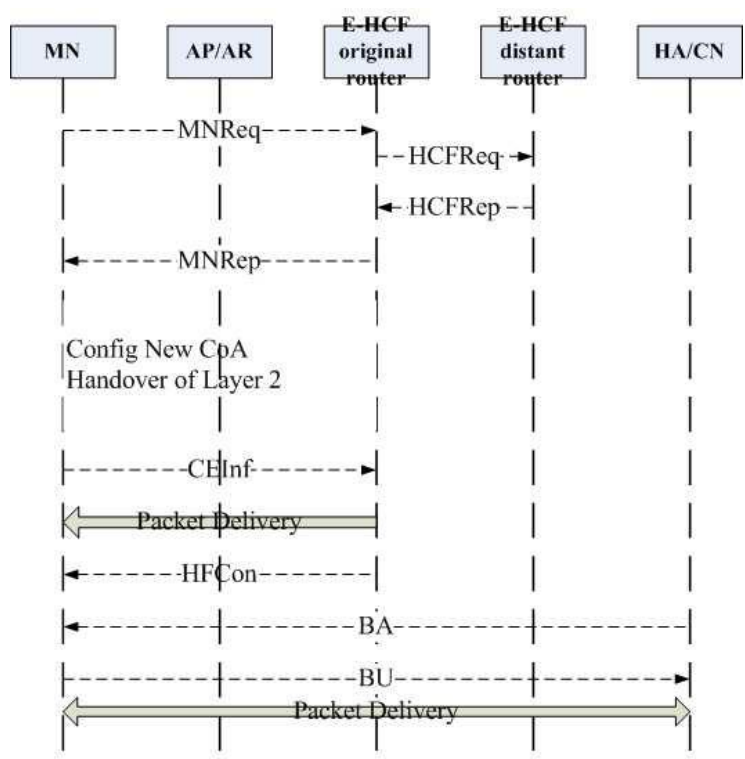

Fig. 3. E-HCF Procedure (E-HCF original router is an attached router with an E-HCF function; the E-HCF distant/remove router is a router with who an E-HCF original router can communicate) 


\section{B. E-HCF Procedure}

We first recall that HCFReq/HCFRep messages are used between $E-H C F$ routers for extra-domain handovers. Each $E$ $H C F$ router must record and update its database periodically. This database helps to decide an unique new IP configuration in order to adapt for $M N$ movements without the $D A D$ phase during a handover.

As illustrated on Figure 3, the $E-H C F$ procedure is composed of the following steps:

- Moving in the network, if the threshold of the received signal strength is overstepped, the $M N$ begins to probe the neighbor $A R / A \mathrm{P}$ 's information, including the signal strength, some IP addresses, $A P$ 's $B S S I D s, A R$ interface addresses and the sub-network prefix. Then the $M N$ sends a MNReq message to its $E-H C F$ original router (via its $A R / A P)$ to report this information.

- Receiving the MNReq message, the AR stops to forward all the packets sent to the $M N$ and forwards them to its $E-H C F$ original router in order to avoid the packet loss during the handover procedure.

- Receiving the MNReq message, the E-HCF original router decides to which $A R / A P$ the $M N$ will be associated. The choice of the $A R / A P$ is mostly based on database obtained with periodic exchange messages from an $E$ $H C F$ router to another (HCFReq and HCFRep messages) or with periodic exchange messages from ARs/APs/MNs. For example, if the number of registered $M N \mathrm{~s}$ in one $A R$ or $A P$ has reached a limit, the $E-H C F$ original router will not attach the $M N$ to this saturated $A R$ or $A P$. After making the previous decision, the $E-H C F$ original router sends to the $M N$ a MNRep message which consists of a new $A P$ 's $B S S I D$, an $A R$ interface address, a sub-network prefix and a new IP address.

- With the MNRep message, the $M N$ can obtain its new CoA and configure it automatically.

- The $M N$ sends a CEInf message to its $E-H C F$ original router to confirm its new attachment.

- After receiving the CEInf message, the $E-H C F$ original router transfers the buffered packets to the $M N$ 's new CoA. Then, the E-HCF original router sends an HFCon message to end the handover procedure.

- The $M N$ can then exchange Binding Update $(B U)$ and Binding Acknowledgement $(B A)$ messages with its home agent and its correspondent node.

As shown in the $E-H C F$ procedure, a $M N$ can obtain its new $C O A$ before it really attaches to its next $A R / A P$. Moreover, any $D A D$ latency (about $1000 \mathrm{~ms}$ ) is avoided. Thus, the $E-H C F$ approach allows the reduction of both the traditional handover latency and the packet loss. The handover performance is thus optimized compared to a traditional approach.

\section{E-HCF PERFORMANCE ESTIMATION}

The $E-H C F$ performance estimation has been evaluated in terms of the total handover latency and of the packet loss with an analytical model. This model allows us to compare our $E-H C F$ handover with the standard handover of the MIPv6 protocol.

\section{A. E-HCF Latency Analysis}

According to the handover procedure on Figure 3 , we cite the following latency notations to estimate the handover latency:

- LEHCF: Total handover latency with the E-HCF approach.

- Lscan: Latency due to the MN's original scanning of its neighbour $A R / A P$ 's information.

- LMNReq: Latency for a $M N$ to send a MNReq message to its $E-H C F$ original router.

- $L_{d e c}$ : Latency necessary to an $E-H C F$ router to decide which $A R / A P$ the $M N$ should be attached (including the short delays to send an HCFReq message and to receive an HCFRep message).

- LMNRep: Latency for an E-HCF router to send a MNRep message to the $M N$.

- LCNinf: Latency necessary for a $M N$ to auto-configure its new $C o A$.

- $L_{\text {conf }}$ : Latency due to the fact that an $E-H C F$ router sends buffered packets and a HFCon message.

- $L_{B U / B A}$ : Binding Update/Binding Acknowledgement latency.

The overall $E-H C F$ handover latency $L_{E H C F}$ can be summed as following:

$$
\begin{aligned}
& L_{E H C F}= \\
& \quad L_{\text {scan }}+L_{\text {MNReq }}+L_{d e c}+L_{\text {MNRep }}+ \\
& L_{C N i n f}+L_{\text {conf }}+L_{B U / B A}
\end{aligned}
$$

As this $L_{E H C F}$ depends upon the mobile link bandwidth and the computation capacity of each entity in the wireless network, we summarize the parameter values used in our numerical analysis in Table I.

TABLE I

Parameter Setting

\begin{tabular}{|l|l|l|}
\hline Parameter & Value & Comment \\
\hline Channel scan time & $50 \mathrm{~ms}$ & MIPv6 standard \\
\hline BU/BA latency & $140 \mathrm{~ms}$ & MIPv6 standard \\
\hline Wireless link bandwidth & $5.5 \mathrm{Mb} / \mathrm{s}$ & IEEE $802.11 \mathrm{~b}$ \\
\hline Wireless link bandwidth & $2 \mathrm{Mb} / \mathrm{s}$ & UMTS \\
\hline Wireless link bandwidth & $150 \mathrm{~kb} / \mathrm{s}$ & GPRS \\
\hline Wireless link bandwidth & $9 \mathrm{~kb} / \mathrm{s}$ & GSM \\
\hline AR computation capacity & $20 \mathrm{Mb} / \mathrm{s}$ & general router \\
\hline MN computation capacity & $10 \mathrm{Mb} / \mathrm{s}$ & PC computation capacity \\
\hline MNReq message size & $72 \mathrm{byte}$ & E-HCF approach \\
\hline MNRep message size & 45 byte & E-HCF approach \\
\hline HCFReq message size & 45 byte & E-HCF approach \\
\hline HCFReq message size & 45 byte & E-HCF approach \\
\hline CEInf message size & 45 byte & E-HCF approach \\
\hline HFCon message size & 24 byte & E-HCF approach \\
\hline
\end{tabular}

\section{B. Numerical Results of the Total E-HCF Latency}

With the parameters of Table I, we give a latency comparison between the standard handover latency and the $E$ $H C F$ latency according to equation (1). These latencies are functions of the wireless link bandwidths (WiFi, UMTS, GPRS and GSM) and of the computation capacity. For example, the 
$L_{M N R e q}$ latency can be numerically estimated as following: with a $10 \mathrm{Mb} / \mathrm{s}$ computation capacity, a $M N$ needs $57.6 \mu \mathrm{s}$ to generate a 72-byte MNReq message, whereas, $28.8 \mu$ s are required for an Access Router. Putting this 72-byte message on a $9 \mathrm{~kb} / \mathrm{s}$ GSM network, requires about $64 \mathrm{~ms}$, so that the global of $L_{M N R e q}$ is about $64 \mathrm{~ms}$.

On Figure 4, the standard MIPv6 handover latency (1290 $\mathrm{ms}$ ) is the first figure displayed on the left. The rest of the figures are the $E-H C F$ handover latencies based on $\mathrm{WiFi}$, UMTS, GPRS and GSM link bandwidths. We note that the various $E-H C F$ latencies are not really different when link bit rates vary from $150 \mathrm{~kb} / \mathrm{s}$ to $5.5 \mathrm{Mb} / \mathrm{s}$. If the link bit rate drops to $9 \mathrm{~kb} / \mathrm{s}$ (GSM), the $E-H C F$ handover latency raises up to 450 ms. As a result, the wireless link bandwidth has an important influence over the overall handover procedure. Let us focus on the $E-H C F$ latency with the IEEE $802.11 \mathrm{~b}$ wireless networks. The average of the $E-H C F$ handover latency is about $200 \mathrm{~ms}$. This value of $200 \mathrm{~ms}$ is validated by our simulation results on OPNET illustrated on Figure 5.

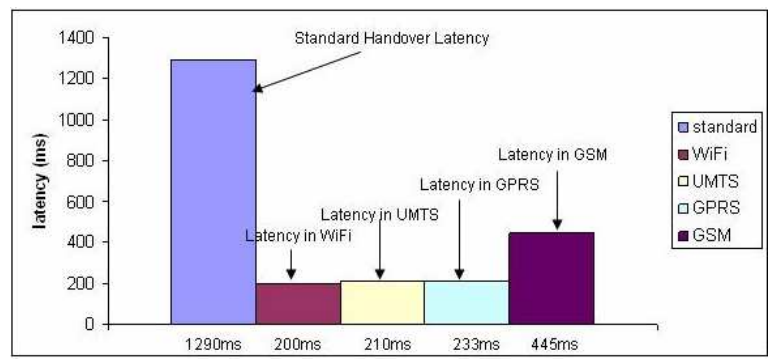

Fig. 4. E-HCF handover latencies as a function of wireless link bandwidths

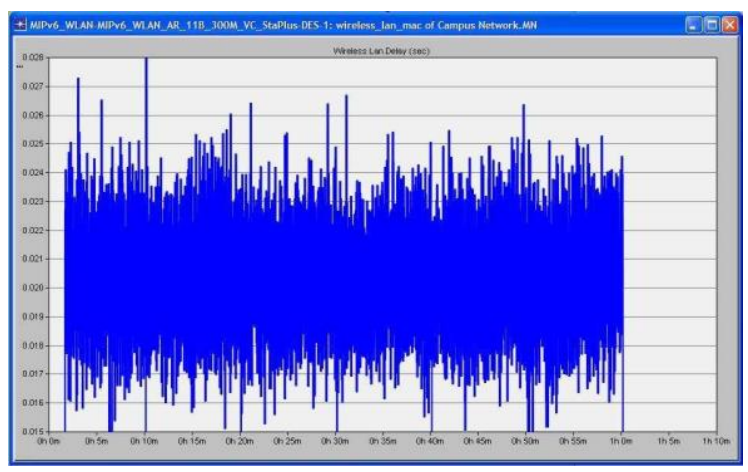

Fig. 5. E-HCF handover latency by simulation

Although the latency reduction from $1290 \mathrm{~ms}$ to $200 \mathrm{~ms}$ is significant, the value of $200 \mathrm{~ms}$ is still too long to support a real time application in wireless networks. This is due to the number of channel scans. As a results, we propose a fast $E-H C F$ method in which a $M N$ can immediately request its $E-H C F$ router without probing for the connection information, if the threshold of the received signal strength is overstepped. The $E-H C F$ router then decides the next attached point. Our simulation results show that the average of the fast E-HCF latency can drop to $100 \mathrm{~ms}$.

\section{E-HCF Loss}

In terms of packet loss with the $E-H C F$ approach, packets can be stored into a buffer during the handover (see subsection II-B). Figure 6 illustrates the comparison of packet loss rates between the E-HCF approach and the MIPv6 standard. The upper curve represents the number of lost packets with the MIPv6 standard (38 packets received out of 100 emitted packets), whereas the bottom curve with E-HCF approach (68 packets received out of 100 emitted packets). This gives a typical 30\% gain with the E-HCF approach.

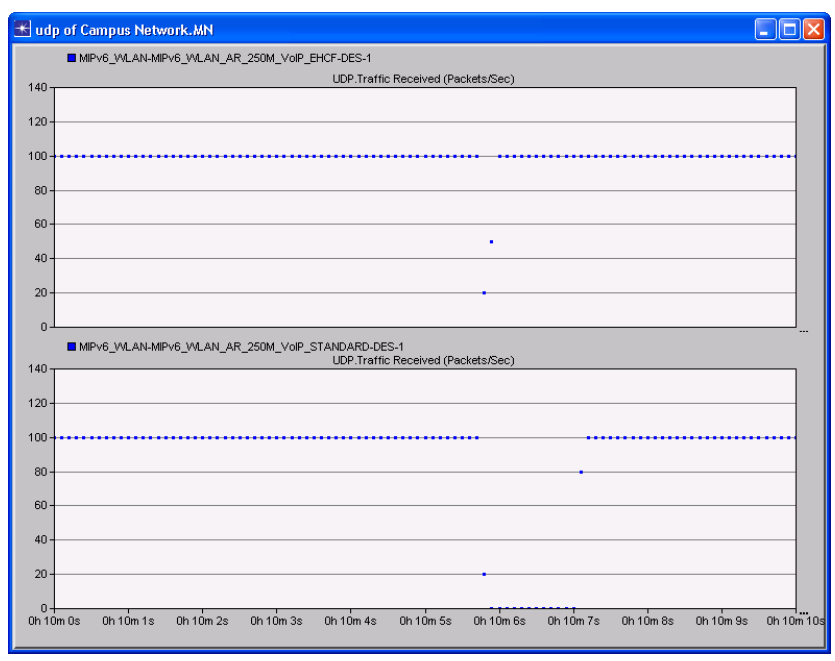

Fig. 6. Comparison of loss rates between the E-HCF approach and the MIPv6 standard by simulation

\section{CONCLUSION}

In order to improve the handover performance for the Mobile IPv6, this paper studies an original E-HCF approach which allows to collect and store some link and network data. Regarding the classical Mobile IPv6 handover performance, our numerical results validated by simulations show that the $E-H C F$ approach enables to decrease both the total handover latency and the packet loss significantly.

We focused on the handover performance at the Network Layer. We now are interested to also improve the handover performance at the Link Layer with a "graph" solution. Our future goal aims at improving the handover performance both at the Network Layer for the Mobile IPv6 and at the Link Layer for IEEE 802.11 networks with a cross-layer proposal.

\section{REFERENCES}

[1] C. Perkins, "IP Mobility support for IPv4," RFC 3220, IETF, January 2002.

[2] D. Johnson, C. Perkins, and J.Arkko, "Mobility Support in IPv6," RFC 3775, June 2004.

[3] G. Z. Wei, A. Wei, K. Xu and H. Deng, "Handover Control Function Based Handover for Mobile IPv6," In Proceedings of Workshop of Evolution towards Next Generation Internet (ICCS 2006), University of Reading, UK, 28-31 May 2006.

[4] C. E. Perkins, Tutorial Mobile Networking through Mobile IPv4, Sun Microsystems, http://www.computer.org/internet/v2n1/ perkins.html. 2005

[5] H. Soliman, C. Castelluccia, K. Malki, and L. Bellier, "Hierarchical Mobile IPv6 mobility management (HMIPv6)," RFC 4140, August 2005. 
[6] H. Fathi, S.H. Shin, K. Kobara, and al, "Leakage-Resilient security Architecture for Mobile IPv6 in Wireless overlay Networks," IEEE Journal on Selected Areas in Communications, vol. 23, No. 11, pp.21822193, November 2005.

[7] Wei Kuang Lai and Jung Chia Chiu, "Improving Handoff Performance in Wireless Overlay Networks by Switching between Two-Layer IPv6 and One-Layer IPv6 Addressing," IEEE Journal on Selected Areas in Communications, vol. 23, No. 11, pp. 2129-2137, November 2005.

[8] R. Koodli, Ed. "Fast Handovers for Mobile IPv6," RFC 4068, July 2005.

[9] H. Chaouchi and P. Antunes, "Pre-handover Signaling for QoS Aware Mobility Management," International Journal of Network Management, No. 14, pp.367-374, 2004.

[10] Y. Bi, P. Iyer, "An Integrated IP-layer Handover Solution for Next Generation IP-based Wireless Network", IEEE Vehicular Technology Conference VTC2004-Fall, Vol. 6, pp.3950-3954, Los Angeles, USA, 2004

[11] J. Kristiansson and P. Parnes, "Application-layer Mobility Support for Streaming Real-time Media," Wireless Communications and Networking Conference, Vol.1, pp.268-273, Atlanta, USA, 2004.

[12] S. Kashihara, K. Iida and al, "End-to-End Seamless Handover Using Multi-path Transmission Algorithm," International Conference on Internet Computing 2002, IC'02, pp. 1-7, Las Vegas, USA, 2002.

[13] "IPv6 Address Allocation and Assignment Policy," doc no. ripe267, http://www.ripe.net/ripe/docs/ipv6policy. html,January 2003

[14] "IEEE 802.11f: Recommended Practice for Multi-Vendor Access Point Interoperability via an Inter-Access Point Protocol Access Distribution Systems Supporting IEEE 802.11 Operation", IEEE Standard 802.11, January 2003

[15] Guozhi Wei, "Handover Optimisation using E-HCF Method for the Mobile IPv6 protocol," thesis, University of Paris XII, France, February, 2008.

[16] J. Bournelle and M. Laurent-Marknavicius, "Adaptation et implementation de Diameter/AAA pour Mobile IPv6," http://www-rp.lip6. fr/dnac/5.1-bournelle-article.pdf, 2004

[17] A. Patel, K. Leung et al, "Authentication Protocol for Mobile IPv6," RFC 4285, January, 2006.

[18] J. Arkko, V. Devarappli and F. Dupont, "Using IPSec to Protect Mobile IPv6 Signaling Between Mobile Nodes and Home Agents," RFC 3776, June 2004. 